\title{
Impact of reslizumab on outcomes of severe asthmatic patients: current perspectives
}

This article was published in the following Dove Press journal: Patient Related Outcome Measures

\begin{abstract}
Alicia Padilla Galol,*
Marina Labor ${ }^{2,3, *}$

Angelica Tiotiu ${ }^{4}$

Ilaria Baiardini ${ }^{5}$

Nicola Scichilone ${ }^{6}$

Fulvio Braido ${ }^{7}$

'Unit of Pneumology, Agencia Sanitaria

Costa del Sol, Marbella, Málaga,

Spain; '2Department of Pulmonology,

University Hospital Center Osijek,

Osijek, Croatia; ${ }^{3}$ Faculty of Medicine,

J.J. Strossmayer University of Osijek,

Osijek, Croatia; ${ }^{4}$ Department of

Pulmonology, CHRU Nancy, DevAH

- Development, Adaptation and

Disadvantage, Cardiorespiratory

Regulations and Motor Control,

University of Lorraine, Nancy, France;

${ }^{5}$ Department of Biomedical Sciences,

Humanitas University, Milan, Italy;

${ }^{6}$ Department of Biomedicine and

Internal and Specialistic Medicine

(DIBIMIS), University of Palermo,

Palermo, Italy; ${ }^{7}$ Department of

Internal Medicine, Respiratory

Diseases and Allergy Clinic, University

of Genova - Azienda Policlinico

IRCCs San Martino, Genova, Italy
\end{abstract}

*These authors contributed equally to this work

Correspondence: Angelica Tiotiu Department of Pulmonology, CHRU Nancy, EA 3450 DevAH - Development, Adaptation and Disadvantage, Cardio-

Respiratory Regulations and Motor

Control, University of Lorraine, 9

Avenue de la Forêt de Haye, F-54505 -

Vandoeuvre-les-Nancy, France

Tel +33 $383 \quad 154372$

Fax $+33383 \quad 15354$ I

Email a.tiotiu@chru-nancy.fr

\begin{abstract}
Approximately $5 \%-10 \%$ of asthmatics suffer from severe asthma. New biological treatments represent a great opportunity to reduce asthma burden and to improve asthma patients' lives. Reslizumab will soon be available in several European countries. This anti-IL-5 IgG4/ $\kappa$ monoclonal antibody, administered intravenously at a dose of $3 \mathrm{mg} / \mathrm{kg}$ over 20-50 minutes every 4 weeks, has been shown to be safe and effective in patients with 400 eosinophils $/ \mu \mathrm{L}$ or more in their peripheral blood. The clinical effects in reducing asthma exacerbations and in improving the quality of life and lung function are clear, but further research is needed to determine the best biological compound for a specific cluster of patients. Research data have shown that in patients who were expressing other clinical features of eosinophilic inflammation over asthma (rhinosinusitis and nasal polyposis), the clinical benefit of reslizumab was greater. Furthermore, it has also been observed that in patients with unsatisfactory response to mepolizumab, reslizumab is able to significantly improve the clinical and biological parameters. The aim of personalized medicine is to provide the right drug to the right patient at the right dose at the right moment. The biological treatments that were developed to modify specific pathological pathways not only provide us with the tools for the management of asthma patients but also clarify the biological mechanisms involved in its pathogenesis.
\end{abstract}

Keywords: asthma, patient-reported outcomes, personalized medicine, reslizumab

\section{Reslizumab in the context of personalized medicine of severe asthma}

According to the current estimates, asthma is globally the most prevalent chronic respiratory disease affecting more than 358 million people and its incidence is still increasing. ${ }^{1}$ The introduction of international guidelines (Global Initiative for Asthma, GINA) ${ }^{2}$ for the management of asthma in 1993 and their implementation into clinical practice have not only significantly altered the burden of the disease but also revealed the areas of inadequate treatment control and unmet needs. The significant heterogeneity of asthma leads to the identification of various asthma phenotypes. The problem of severe asthma emerged more clearly when the management strategy introduced the focus on asthma control. ${ }^{3}$ Severe asthma is defined as "asthma which requires treatment with high dose inhaled corticosteroids (ICSs) plus a second controller (and/or systemic corticosteroids) to prevent it from becoming "uncontrolled" or which remains "uncontrolled" despite this therapy". ${ }^{4}$ The prevalence of severe asthma is reported to be around $5 \%$ with high rate of poor control ( $>50 \%$ of patients) with a significantly higher risk of exacerbations (risk ratio 2.59) and a low level of health-related quality 
of life (HRQoL). ${ }^{5}$ Although the prevalence of severe asthma is low, the burden of the disease on the patient, family, health care system, and society is disproportionally high, accounting for up to $30 \%$ of all costs incurred by all asthmatic patients. ${ }^{6}$ Solving the problem of severe asthma is complicated due to the fact that it is a heterogeneous condition presenting with different phenotypes that are based on distinct endotypes. ${ }^{4}$ Various studies have identified several phenotypes of severe asthma primarily based on a different set of input parameters, but $\sim 50 \%$ of severe asthma patients have elevated eosinophil levels and certain level of resistance to corticosteroid treatment. Both these characteristics are associated with increased burden (lower rate of control, higher rate of exacerbations, and adverse events) and costs of asthma (>double). ${ }^{7}$ Based on this fact, a corticosteroid sparing strategy that would downregulate the high level of eosinophils could produce benefits on disease control and reduce its burden. Both the innate and adaptive immunity pathways of type-2 airway inflammation ("Th2-high asthma" phenotype) are involved with many cell types and signaling molecules. ${ }^{8}$ In severe eosinophilic asthma, interleukin-5 (IL-5) has been found to be one of the major players in the recruitment of eosinophils; therefore, biological drug-targeting strategies have been developed by using humanized monoclonal antibodies (hmAbs). Of the two hmAbs that have been created, one binds to IL-5 preventing from binding to its receptor (mepolizumab and reslizumab) and the other (benralizumab) binds to IL-5 alpha chain receptor (IL-5R $\alpha$ ). Both mepolizumab and reslizumab have been shown to be clinically effective, allowing for a precision medicine approach targeting a specific pathway. ${ }^{9}$ Nowadays, the use of novel research technologies (genomics, transcriptomics, proteomics, and metabolomics) allows us to define heterogeneity among patient subgroups and among individual patients on the basis of a specific pathophysiological patterns, networks of effector cells, and signaling molecules, allowing us to specifically target these pathways. Although it might seem that precision and personalized medicine are synonyms, this is not the case. Unlike precision medicine that targets specific endotypes or major endotypes behind specific phenotypes in a (sub)group of patients, personalized medicine is an individually tailored treatment. Meanwhile, specific markers like blood and sputum eosinophils, fraction of exhaled nitrous oxide, periostin, and even recent omics approaches allow only a precision medicine approach. ${ }^{10,11}$ As with other medicines, an individual response to different drugs of the same class is to be expected. Strategies to decode the pathophysiological mechanisms behind the specific phenotype in individual patients using the newest omics sciences will allow a personalized approach to individual patients. ${ }^{12,13}$ However, to develop such strategies, we would need the records of patients who were treated with specific biological agents, like reslizumab, supported with biobanks and high-throughput omics technologies.

\section{Biological properties of anti-IL-5 molecules}

Reslizumab is a humanized $\mathrm{IgG} 4 / \mathrm{\kappa} \mathrm{mAb}$ produced by means of a synthetic process based on recombinant technology, which allows the integration of the rat antigen recognition sites for human IL-5 to a human IgG4. ${ }^{14-16}$ The antibody can bind free IL-5 molecules (dissociation constant $=81 \mathrm{pM}$; association constant of $4.9 \times 105 \mathrm{per} \mathrm{M} / \mathrm{s}$ ). This bonding prevents IL-5 from linking to its receptor. ${ }^{16-19}$ It is matter of fact that reslizumab at a concentration of $0.5 \mathrm{nM}$ inhibits IL-5 binding by $50 \%$ and at $45 \mathrm{nM}$ inhibits IL-5-induced proliferation by $50 \%$. Reslizumab has been tested in animal models featuring eosinophilic inflammation, ${ }^{20}$ such as allergic mice and ascaris-responsive monkeys. The effect of the drug lasted for 6 months after administration in monkeys, thereby reducing the ascaris challenge-induced eosinophilia by $75 \% .{ }^{21}$ In ovalbumin-sensitized guinea pig model, reslizumab $(0.03 \mathrm{mg} /$ $\mathrm{kg}$ and $1 \mathrm{mg} / \mathrm{kg}$ ) administered 2 hours before the specific challenge reduced eosinophilia, airway hyperreactivity, and bronchoconstriction. In ovalbumin-sensitized rabbits, reslizumab decreased the eosinophil influx into the skin without impacting the overall number of cells in the skin. ${ }^{16}$ The aforementioned data justify the in vivo investigation that demonstrated that reslizumab reduces airway hyperresponsiveness and inhibits the influx of eosinophils into the lungs. For example, in animals exposed to an agent that increases the eosinophil count to 298,000 cells $/ \mathrm{mL}$, a $1 \mathrm{mg} / \mathrm{kg}$ dose reduced eosinophil in the bronchoalveolar lavage counts to 108,000 cells $/ \mathrm{mL}^{16}$ Furthermore, in the Phase II Res-5-0010 study, reslizumab significantly lowered the active airway eosinophilic inflammation by reducing the sputum eosinophil count by $95 \%$ compared with a $39 \%$ reduction for placebo. ${ }^{22}$ At present, the anti-IL-5 $\mathrm{mAb}$ families also include mepolizumab, while benralizumab binds to the surface of IL-5 receptor. Mepolizumab, unlike reslizumab, is a humanized IgG1. Mepolizumab is composed of 4 light and heavy chains linked by a disulfide bridge. The antibody binds with high-specificity and -affinity IL-5 ${ }^{23}\left(\mathrm{IC}_{50}\right.$ $<1 \mathrm{nM} ; K_{\mathrm{d}}=4.2 \mathrm{pM}$ ) and avoids binding to IL-5R $\alpha .^{24,25}$ Both mepolizumab and reslizumab bind to epitopes within the IL-5R $\alpha$-binding domain, while mAbs interacting with IL-5 $\beta \mathrm{c}$ domain are currently not available, although this may represent a potential target. 


\section{Functional and biological results from pivotal trials}

Reslizumab has been recently approved for the treatment of severe eosinophilic asthma. This is due to its safety profile and efficacy. The first study that showed the efficacy of the drug in reducing eosinophils in asthmatics was conducted by Kips et al. ${ }^{26}$ The pilot investigation was carried out in a small number of subjects $(\mathrm{n}=32)$ who were suffering from severe persistent asthma despite high-dose ICSs or systemic corticosteroids and they were treated with placebo or reslizumab intravenously (IV). No significant effect on eosinophil counts was observed in patients treated with lower doses, whereas a sustained response was observed in those treated with the highest dose. Interestingly, there were no significant differences in lung function or symptom scores among the study groups. These disappointing findings can be reasonably explained by the "small and not powered" study population and by the lack of preselection of patients with eosinophilia.

To overcome this issue, a multicenter, randomized, double-blind, placebo-controlled study was designed to explore the effects of reslizumab versus placebo in poorly controlled adult asthmatics with induced sputum eosinophils of $3 \%$ or more..$^{22}$ As expected, patients who received reslizumab had a significant decrease in sputum eosinophils. An improvement from baseline in forced expiratory volume in 1 second (FEV1) was found in the reslizumab group compared with placebo. In addition, a trend for improvement in asthma control and reduction in exacerbation rates was shown in the active group, although it was not statistically significant.

Two subsequent studies had the merit of including blood eosinophil count instead of sputum eosinophils to define eosinophilic asthma, thus limiting the intrinsic variability due to the difficulties in collecting sputum from severe asthmatics and lack of reproducible findings. Two other trials assessed the efficacy of reslizumab in severe asthmatics. ${ }^{27} \mathrm{~A}$ total of 953 patients received reslizumab at a dose of $3 \mathrm{mg} / \mathrm{kg}$ or placebo IV every 4 weeks (Q4W) for a total of 13 doses. Patients in the active group showed early improvements in lung function and in symptoms, as well as a reduction in asthma exacerbations compared with placebo. Corren et $\mathrm{al}^{28}$ investigated the efficacy of reslizumab in a cohort of 496 uncontrolled asthmatic subjects with a wide range of blood eosinophilic concentrations. The efficacy of reslizumab in terms of improvements in pulmonary function, as well as in terms of Asthma Control Questionnaire (ACQ) scores and rescue inhaler use, was detected only in subgroup analyses in subjects with eosinophil counts $>400 / \mathrm{L}$.

\section{Patient-reported outcomes (PROs) results from pivotal trials}

PROs - "any report coming from patients about a health condition that comes directly from the patient, without interpretation of the patient's response by a clinician or anyone else" 29 - allow to capture a patient's subjective perspective on the effectiveness of a treatment. As such, PROs provide the possibility to include the input coming from patients in assessing the impact of a treatment and may personalize the treatment decision-making process..$^{30}$ All the registered studies evaluating the efficacy and safety of reslizumab in asthma patients include the assessment of PROs as primary ${ }^{22}$ or secondary outcomes. ${ }^{29-31}$ In the study by Castro et al, ${ }^{22}$ the primary end point was the change of asthma control (assessed by ACQ-7) ${ }^{32}$ in patients who were treated with reslizumab or placebo for 15 weeks. The mean change in ACQ-7 score from baseline to the study end was modest and did not achieve statistical significance ( -0.7 in the reslizumab group and -0.3 in the placebo group, $p=0.0541)$. A subgroup analysis of patients with comorbid nasal polyps detected a significantly greater improvement in ACQ-7 scores in the active group than in placebo group $(-1.0 \mathrm{vs}-0.1, p=0.0119)$. The minimal important difference (MID) of $0.5,{ }^{33}$ which represents the smallest change perceived as beneficial by patients, ${ }^{34}$ was achieved in $59 \%$ of patients in the reslizumab group and in $40 \%$ of patients in the placebo group (odds ratio: 2.06, $p=0.0973$ ). The results of the two parallel studies described by Hart et $\mathrm{a}^{25}$ showed that the significant effect of reslizumab on the primary outcome (frequency of asthma exacerbations) moves in parallel with the effect on PROs. As a matter of fact, the scores of Asthma Quality of Life Questionnaire (AQLQ), ${ }^{35}$ ACQ-7, ${ }^{32}$ and Asthma Symptom Utility Index $(\mathrm{ASUI})^{36}$ show a significantly greater improvement $(p<0.05)$ in the active group compared with placebo. Moreover, compared to placebo group, a higher percentage of patients in the reslizumab group reached the MID ${ }^{37}$ in AQLQ (study 1: $74 \%$ vs $65 \%, p=0.03$; study $2: 73 \%$ vs $62 \%, p=0.02$ ) and in ACQ-7 (study 1: $76 \%$ vs $63 \%, p=0.0002$; study $2: 77 \%$ vs $61 \%, p=0.0002)$. Corren et a ${ }^{28}$ showed that the mean change of ACQ-7 score was modest from baseline to the end of the study (week 16) and the differences between reslizumab and placebo groups did not reach statistical significance. However, the percentage of patients who reached the MID was significantly higher with reslizumab than with placebo ( $71 \%$ vs $57 \%, p=0.01)$. Moreover, a secondary analysis in the subgroup of patients with a blood eosinophil count of $\geq 400$ cells $/ \mathrm{mL}$ detected a small but significant improvement 
with reslizumab treatment compared with placebo treatment $(0.272$ vs $0.002, p=0.0436)$, corresponding to the improvement in FEV1. Both reslizumab $0.3 \mathrm{mg} / \mathrm{kg}$ and 3.0 $\mathrm{mg} / \mathrm{kg}^{29}$ significantly improved asthma control (ACQ-7) and frequency and severity of symptoms (ASUI) compared with placebo. Both the tools detected a greater magnitude of improvement in the group treated with reslizumab $3 \mathrm{mg} /$ $\mathrm{kg}$. At the end of the study, MID of ACQ-7 was reached by a similar percentage of patients in the reslizumab and placebo groups, without any significant difference among the groups. Improvements in AQLQ scores versus placebo were observed for reslizumab $3 \mathrm{mg} / \mathrm{kg}(1.138$ vs $0.779, p=0.0241)$ but not for reslizumab $0.3 \mathrm{mg} / \mathrm{kg}$ (1.057 vs $0.779, p=0.0822)$. A greater proportion of patients in the active groups compared to placebo group achieved the MID of AQLQ at the study end. The difference versus placebo was significant for reslizumab $3 \mathrm{mg} / \mathrm{kg}(64 \%$ vs $48 \%, p=0.0189)$ but not for reslizumab $0.3 \mathrm{mg} / \mathrm{kg}(59 \%$ vs $48 \%, p>0.05)$.

\section{Placement of reslizumab in biological treatment of asthma}

Reslizumab administered by IV perfusion has demonstrated a notable effect, compared to placebo, in reducing exacerbations and in triggering significant improvements in pulmonary function in adult patients with severe eosinophilic asthma (baseline levels of eosinophils $\geq 400$ cells $/ \mu \mathrm{L}$ ) that remains inadequately controlled despite being treated with high doses of ICS + long-acting beta-agonists and/or oral corticoids. ${ }^{22,27-29}$ It should be noted that clinical trials on mepolizumab and reslizumab have produced similar results, but the studied populations have never been exactly the same. However, in the absence of any direct comparisons, it is impossible to establish the differences (or otherwise) between these two drugs, although reslizumab, unlike subcutaneous mepolizumab, could be limited by the need for IV administration but could provide a greater sense of care to the patient. Moreover, no specific studies have confirmed the effect of reslizumab on reducing the use of oral corticoids, whereas these data are available in the case of mepolizumab. ${ }^{38,39}$ These limitations must be considered before selecting treatment. More recently, however, a study investigated 10 prednisone-dependent asthmatics (blood eosinophils $>300$ cells $/ \mu \mathrm{L}$ and sputum eosinophils $>3 \%$ ) who had previously received mepolizumab (100 mg subcutaneous dose Q4W) for at least 1 year and two infusions of placebo (Q4W) followed by 4 infusions of $3 \mathrm{mg} /$ $\mathrm{kg}$ reslizumab $\mathrm{Q} 4 \mathrm{~W}$, in a single-blind, placebo-controlled sequential trial. ${ }^{40}$ The authors found that the weight-adjusted IV reslizumab was superior to the fixed-dose subcutaneous mepolizumab in attenuating eosinophilia. Attenuation of both local and systemic eosinophilia was associated with statistically significant improvements in asthma control and FEV1. Reslizumab could, therefore, be also used as an alternative for those patients who show no improvement with mepolizumab. In 2016, Froidure et $\mathrm{al}^{41}$ created a decision chart to aid the selection of appropriate biological treatment, based on the levels of $\operatorname{IgE}$ and eosinophilia in the blood, but there is currently some controversy concerning the mAb that should be first used in case of allergic and eosinophilic asthma. Some authors have suggested that omalizumab should be used as the first option in patients with an allergic phenotype, regardless of the levels of eosinophils in the peripheral blood, with anti-IL-5 as an alternative for allergic patients with blood eosinophilia in whom omalizumab treatment has failed. ${ }^{42} \mathrm{~A}$ recently published post-hoc analysis ${ }^{43}$ of two 52 -week placebo-controlled trials of reslizumab IV $3 \mathrm{mg} / \mathrm{kg}$ every 4 weeks in patients with uncontrolled asthma has compared the results for pulmonary function and exacerbations in patients with late-onset asthma to those with early onset asthma. This analysis showed that reslizumab produced greater improvements in lung function and larger reductions in asthma exacerbations in patients with late-onset asthma compared to those with early onset asthma. This could be an important consideration when deciding on biological treatment. Although no studies have been undertaken to compare such findings with the efficacy of omalizumab in patients with late-onset asthma, the results of the aforementioned analysis suggest that the use of anti-IL-5 could be posited as a first option for allergic and eosinophilic patients with late-onset asthma. An algorithm for treatment has been created based on phenotypes by Domingo ${ }^{42}$ and Alvarez et $\mathrm{al},{ }^{44}$ but the value of these approaches needs to be explored in real life. Other factors that possibly need to be considered before choosing a mAb are asthma severity and pulmonary function. A recent study has published pooled data from duplicate, Phase III, reslizumab versus placebo trials, in which patients were categorized according to severity (Steps 4 and 5 of the GINA guidelines). This study showed that, compared with placebo, reslizumab has increased FEV1 in Step 4 and Step 5 groups by $103 \mathrm{~mL}(52-154 \mathrm{~mL})$ and $237 \mathrm{~mL}$ (68-407 mL), respectively. Additionally, reslizumab reduced exacerbation rates by $53 \%(0.36-0.62)$ and $72 \%(0.15-0.52)$ in Step 4 and Step 5 groups, respectively. ${ }^{45}$ Furthermore, reslizumab has been shown to improve nasal polyposis in patients with severe asthma. ${ }^{46}$ Some meta-analyses have also demonstrated the beneficial effects of anti-IL-5 in patients with refractory polyposis and of omalizumab in patients with polyposis and severe asthma. ${ }^{47}$ No studies have yet directly 
compared the effects of reslizumab on nasal polyposis with that of other anti-IL-5 or omalizumab, and so specific studies are required to identify the most suitable first-choice treatment in this respect.

\section{Is there a need for new data?}

The introduction of specific biological therapies, such as anti-IgE and anti-IL-5 antibody treatments, at Step 5 of the GINA guidelines has opened a new era of precision medicine in asthma since these agents targeted specific severe asthma pathways, either allergic or eosinophilic. ${ }^{48}$ The IL-5 neutralizing antibodies mepolizumab and reslizumab were licensed in the USA and Europe following successful Phase III studies that demonstrated reduced asthma exacerbations frequency and improved baseline airflow obstruction ${ }^{27,49}$ in patients with high blood eosinophil levels. Another immunomodulator that mediates the effect of IL-5 is benralizumab, an antibody directed against IL-5R $\alpha$, which showed beneficial effects on exacerbations and lung function, particularly in those with increased blood eosinophil count. ${ }^{50}$ Unfortunately, currently, we do not have a comparative study of these biological therapies in terms of effectiveness and tolerability. A recent Cochrane meta-analysis ${ }^{51}$ including 13 studies on 6000 participants ( 4 with mepolizumab, 4 with reslizumab, and 5 with benralizumab) supports the use of anti-IL-5 treatments as an adjunct to standard therapies in patients with severe eosinophilic asthma and poor control on the basis that these treatments roughly halve the rate of asthma exacerbations in this population. However, the authors concluded that there is limited evidence for improved HRQoL scores and lung function in patients treated by anti-IL-5. The safety profile of antiIL-5 treatment is acceptable since and high rate of serious adverse events has not been registered. In this meta-analysis, the number of adverse events leading to discontinuation with mepolizumab or reslizumab was similar to placebo, but significantly higher compared to placebo when benralizumab was interrupted (36/1599 benralizumab vs 9/998 placebo). A possible explanation may be that mepolizumab and reslizumab markedly decrease blood eosinophils but a small number remains, whereas benralizumab almost completely deplete the eosinophils. The implications for efficacy and/ or adverse events are still unclear, although benralizumab data are intriguing. ${ }^{51}$ Some relevant results are also available on dupilumab. This mAb is directed against the $\alpha$-subunit of IL-4 receptor that can block signaling from IL-4 and IL-13. It is shown that dupilumab given subcutaneously can increase lung function and reduce severe exacerbations in patients with uncontrolled persistent asthma, even in patients with low blood eosinophil levels. The transient increase of blood eosinophil during the treatment with this drug needs to be further investigated. ${ }^{52}$ Post-licensing real-life studies are needed to establish the effectiveness and tolerability of these therapies, their optimal duration, long-term benefits and adverse effects, risk of relapse on withdrawal, and the effect in non-eosinophilic patients and children (particularly under 12 years). The comparison of various anti-IL-5 treatments with anti-immunoglobulin E (omalizumab) in patients eligible for both is needed. For benralizumab, future studies should be undertaken to establish the rates of adverse events prompting discontinuation. ${ }^{51,53}$ In the new era of precision medicine in severe asthma, there is an increasing need for developing biomarkers that will guide clinicians in the management of asthma. For the clinician to understand how to select the right therapy for the right patient, we also need more phenotypic and predictive biomarkers for assessing the treatment response. For anti-IL-5 therapies, we probably need to look beyond elevated blood eosinophil counts as a predictive factor.

\section{Disclosure}

The authors report no conflicts of interest in this work.

\section{References}

1. GBD 2015 Chronic Respiratory Disease Collaborators. Global, regional, and national deaths, prevalence, disability-adjusted life years, and years lived with disability for chronic obstructive pulmonary disease and asthma, 1990-2015: a systematic analysis for the Global Burden of Disease Study 2015. Lancet Respir Med. 2017;5(9):691-706.

2. Global Initiative for Asthma (GINA) [homepage]. Available from: http:// ginasthma.org/. Accessed February 1, 2018

3. Bateman ED, Hurd SS, Barnes PJ, et al. Global strategy for asthma management and prevention: GINA executive summary. Eur Respir J. 2008;31(1):143-178.

4. Chung KF, Wenzel SE, Brozek JL, et al. International ERS/ATS guidelines on definition, evaluation and treatment of severe asthma. Eur Respir J. 2014;43(2):343-373.

5. Larsson K, Ställberg B, Lisspers K, et al. Prevalence and management of severe asthma in primary care: an observational cohort study in Sweden (PACEHR). Respir Res. 2018;19(1):12.

6. Chastek B, Korrer S, Nagar SP, et al. Economic burden of illness among patients with severe asthma in a managed care setting. J Manag Care Spec Pharm. 2016;22(7):848-861.

7. Lefebvre P, Duh MS, Lafeuille MH, et al. Burden of systemic glucocorticoid-related complications in severe asthma. Curr Med Res Opin. 2017;33(1):57-65.

8. King GG, James A, Harkness L, Wark PAB. Pathophysiology of severe asthma: we've only just started. Respirology. 2018;23(3):262-271.

9. Canonica GW, Senna G, Mitchell PD, O’Byrne PM, Passalacqua G, Varricchi G. Therapeutic interventions in severe asthma. World Allergy Organ J. 201;9(1):40.

10. Richards LB, Neerincx AH, van Bragt JJMH, Sterk PJ, Bel EHD, Maitlandvan der Zee AH. Biomarkers and asthma management: analysis and potential applications. Curr Opin Allergy Clin Immunol. 2018;18(2):96-108.

11. Svenningsen S, Nair P. Asthma endotypes and an overview of targeted therapy for asthma. Front Med. 2017;4:158. 
12. Canonica GW, Ferrando M, Baiardini I, et al. Asthma: personalized and precision medicine. Curr Opin Allergy Clin Immunol. 2018;18(1): 51-58.

13. Godar M, Blanchetot C, de Haard H, Lambrecht BN, Brusselle G. Personalized medicine with biologics for severe type 2 asthma: current status and future prospects. MAbs. 2018;10(1):34-45.

14. Chung KF. Targeting the interleukin pathway in the treatment of asthma. Lancet. 2015;386(9998):1086-1096.

15. Walsh GM. Profile of reslizumab in eosinophilic disease and its potential in the treatment of poorly controlled eosinophilic asthma. Biologics. 2013;7:7-11.

16. Egan RW, Athwal D, Bodmer MW, et al. Effect of Sch 55700, a humanized monoclonal antibody to human interleukin-5, on eosinophilic responses and bronchial hyperreactivity. Arzneimittelforschung. 1999;49(9):779-790.

17. Cardet JC, Israel E. Update on reslizumab for eosinophilic asthma. Expert Opin Biol Ther. 2015;15(10):1531-1539.

18. Lim H, Nair P. Efficacy and safety of reslizumab in patients with moderate to severe eosinophilic asthma. Exp Rev Respir Med. 2015;9(2): 135-142.

19. Zhang J, Kuvelkar R, Murgolo NJ, et al. Mapping and characterization of the epitope(s) of Sch 55700, a humanized mAb, that inhibits human IL-5. Int Immunol. 1999;11(12):1935-1944.

20. Walsh GM. Reslizumab, a humanized anti-IL-5 mAb for the treatment of eosinophil-mediated inflammatory conditions. Curr Opin Mol Ther. 2009;11(3):329-336.

21. Egan RW, Athwahl D, Chou CC, et al. Inhibition of pulmonary eosinophilia and hyperreactivity by antibodies to interleukin-5. Int Arch Allergy Immunol. 1995;107(1-3):321-322.

22. Castro M, Mathur S, Hargreave F, et al; Res-5-0010 Study Group. Reslizumab for poorly controlled, eosinophilic asthma: a randomized, placebo-controlled study. Am J Respir Crit Care Med. 2011;184(10): 1125-1132.

23. Zia-Amirhosseini P, Minthorn E, Benincosa L, et al. Pharmacokinetics and pharmacodynamics of SB-240563, a humanized monoclonal antibody directed to human interleukin-5, in monkeys. J Pharmacol Exp Ther. 1999;291(3):1060-1067.

24. Lopez A, Sanderson C, Gamble J, Campbell H, Young I, Vadas M. Recombinant human interleukin 5 is a selective activator of human eosinophil function. J Exp Med. 1988;167:219-224.

25. Hart TK, Cook R, Zia-Amirhosseini P, et al. Preclinical efficacy and safety of mepolizumab (SB-240563), a humanized monoclonal antibody to IL-5, in cynomolgus monkeys. J Allergy Clin Immunol. 2001;108(2): 250-257.

26. Kips JC, O'Connor BJ, Langley SJ, et al. Effect of SCH55700, a humanized anti-human interleukin-5 antibody, in severe persistent asthma: a pilot study. Am J Respir Crit Care Med. 2003;167(12):1655-1659.

27. Castro M, Zangrilli J, Wechsler ME, et al. Reslizumab for inadequately controlled asthma with elevated blood eosinophil counts: results from two multicentre, parallel, double-blind, randomized, placebo-controlled, phase 3 trials. Lancet Respir Med. 2015;3(5):355-366.

28. Corren J, Weinstein S, Janka L, Zangrilli J, Garin M. Phase 3 study of reslizumab in patients with poorly controlled asthma: effects across a broad range of eosinophil counts. Chest. 2016;150(4):799-810.

29. Bjermer L, Lemiere C, Maspero J, Weiss S, Zangrilli J, Germinaro M. Reslizumab for inadequately controlled asthma with elevated blood eosinophil levels: a randomized phase 3 study. Chest. 2016;150(4):789-798.

30. Patrick DL, Burke LB, Powers JH, et al. Patient-reported outcomes to support medical product labeling claims: FDA perspective. Value Health. 2007;10(Suppl 2):S125-S137.

31. Corren J, Kavati A, Ortiz B, et al. Patient-reported outcomes in moderate-to-severe allergic asthmatics treated with omalizumab: a systematic literature review of randomized controlled trials. Curr Med Res Opin. 2018;34(1):65-80.
32. Juniper EF, O'Byrne PM, Guyatt GH, et al. Development and validation of a questionnaire to measure asthma control. Eur Respir J. 1999;14(4):902-907.

33. Juniper EF, Svensson K, Mork AC, Stahl E. Measurement properties and interpretation of three shortened versions of the asthma control questionnaire. Respir Med. 2005;99:553-558.

34. Jaeschke R, Singer J, Guyatt GH. Measurement of health status. Ascertaining the minimal clinically important difference. Control Clin Trials. 1989;10(4):407-415.

35. Juniper EF, Guyatt GH, Epstein RS, et al. Evaluation of impairment of health related quality of life in asthma: development of a questionnaire for use in clinical trials. Thorax. 1992;47(2):76-83.

36. Revicki DA, Leidy NK, Brennan- Diemer F, et al. Integrating patient preferences into health outcomes assessment: the multiattribute Asthma Symptom Utility Index. Chest. 1998;114(4):998-1007.

37. Juniper EF, Guyatt GH, Willan A, Griffith LE. Determining a minimal important change in a disease-specific Quality of Life Questionnaire. J Clin Epidemiol. 1994;47:81-87.

38. Bel EH, Wenzel SE, Thompson PJ, et al. Oral glucocorticoid-sparing effect of mepolizumab in eosinophilic asthma. $N$ Engl J Med. 2014;371(13):1189-1197.

39. Lugogo N, Domingo C, Chanez P, et al. Long-term efficacy and safety of mepolizumab in patients with severe eosinophilic asthma: a multicenter, open-label, phase IIIb study. Clin Ther. 2016;38(9):2058-2070.

40. Mukherjee M, Aleman Paramo F, Kjarsgaard M, et al. Weight-adjusted intravenous reslizumab in severe asthma with inadequate response to fixed-dose subcutaneous mepolizumab. Am J Respir Crit Care Med. 2018;197(1):38-46.

41. Froidure A, Mouthuy J, Durham SR, Chanez P, SibilleY, Pilette C. Asthma phenotypes and IgE responses. Eur Respir J. 2016;47(1):304-319.

42. Domingo C. Overlapping effects of new monoclonal antibodies for severe asthma. Drugs. 2017;77(16):1769-1787.

43. Brusselle G, Germinaro M, Weiss S, Zangrilli J. Reslizumab in patients with inadequately controlled late-onset asthma and elevated blood eosinophils. Pulm Pharmacol Ther. 2017;43:39-45.

44. Alvarez FJ, Blanco-Aparicio M, Plaza V, et al. Documento de consenso en asma grave en adultos [Document of consensus on severe asthma in adults]. Monografia de Archivos de Bronconeumología. 2018;5:00158. Spanish.

45. Brusselle G, Canvin J, Weiss S, Sun SX, Buhl R. Stratification of eosinophilic asthma patients treated with reslizumab and GINA Step 4 or 5 therapy. ERJ Open Res. 2017;3(3):pii:00004-2017.

46. Weinstein SF, Germinaro M, Bardin P, Korn S, Bateman ED. Efficacy of reslizumab with asthma, chronic sinusitis with nasal polyps and elevated blood eosinophils. J Allergy Clin Immunol. 2016;AB86:283.

47. Rivero A, Liang J. Anti-IgE and anti-IL5 biologic therapy in the treatment of nasal polyposis: a systematic review and meta-analysis. Ann Otol Rhinol Laryngol. 2017;126(11):739-747.

48. Chung KF. Precision medicine in asthma: linking phenotypes to targeted treatments. Curr Opin Pulm Med. 2017;23:1-7.

49. Ortega HG, Liu MC, Pavord ID, et al. Mepolizumab treatment in patients with severe eosinophilic asthma. N Engl J Med. 2014;371(13): 1198-1207.

50. FitzGerald JM, Bleecker ER, Nair P, et al. Benralizumab, an antiinterleukin-5 receptor $\alpha$ monoclonal antibody, as add-on treatment for patients with severe, uncontrolled, eosinophilic asthma (CALIMA): a randomised, double-blind, placebo-controlled phase 3 trial. Lancet. 2016;388(10056):2128-2141

51. Farne HA, Wilson A, Powell C, Bax L, Milan SJ. Anti-IL5 therapies for asthma. Cochrane Database Syst Rev. 2017;9:CD010834.

52. Bel EH, Ten Brinke. A new anti-eosinophil drugs for asthma and COPD: targeting the trait! Chest. 2017;152(6):1276-1282.

53. Russell R, Brightling CE. Anti-IL-5 for severe asthma: aiming high to achieve success. Chest. 2016;150(4):766-768. 


\section{Publish your work in this journal}

Patient Related Outcome Measures is an international, peer-reviewed, open access journal focusing on treatment outcomes specifically relevant to patients. All aspects of patient care are addressed within the journal and practitioners from all disciplines are invited to submit their work as well as healthcare researchers and patient support groups.
The journal is included in PubMed. The manuscript management system is completely online and includes a very quick and fair peer-review system. Visit http://www.dovepress.com/testimonials.php to read real quotes from published authors.

Submit your manuscript here: http://www.dovepress.com/patient-related-outcome-measures-journal 